\title{
Método para evaluar la alineación de organizaciones públicas de pequeño y mediano porte a la gestión del conocimiento
}

\section{Method for assessing the alignment of small and medium-sized public organizations to knowledge management}

DOI: $10.46814 / \operatorname{lajdv} 3 \mathrm{n} 3-027$

Recebimento dos originais: 01/052021

Aceitação para publicação: 31/06/2021

\author{
José Damião de Melo \\ Doutorando em Difusão do Conhecimento, UFBA. \\ Instituto Federal de Educação, Ciência e Tecnologia de Sergipe, Brasil \\ E-mail:damiao@damiaomelo.com.br \\ Alvaro Luis Henrique Adriazola Uribe \\ Doctorando en Difusión del Conocimiento, UFBA. \\ Universidad Católica de Temuco, Chile. \\ E-mail: alvaro.adriazola.uribe@gmail.com \\ Georgina Ivet Duran Jimenez \\ Doctora em Educação, UFBA. \\ Universidad Católica de Temuco, Chile \\ E-mail: duran.jimenez.georgina@gmail.com

\section{Vania de Jesus} \\ Doutoranda em Ciência da Propriedade Intelectual, UFS. \\ Instituto Federal de Educação, Ciência e Tecnologia de Sergipe, Brasil \\ E-mail: vaniajesus2@gmail.com \\ Valdenice de Jesus Melo \\ Mestre em Educação Profissional e Tecnológica, IFS. \\ Instituto Federal de Educação, Ciência e Tecnologia da Bahia, Brasil. \\ E-mail: nicejesus@gmail.com
}

\section{RESUMEN}

En la tentativa de suplir una importante ausencia en el campo de la gestión del conocimiento en organizaciones de pequeño y mediano porte, presentamos una propuesta de evaluación del nivel de alineación organizacional para la adopción de la Gestión del Conocimiento como elemento gerencial estratégico organizacional, derivado del método OKA. La investigación fue ejecutada en cuatro etapas: las dos primeras envolvieron revisión sistemática de literatura e investigación documental para la formulación de la propuesta del método y, también, para la selección de la entidad a ser estudiada en el estudio de caso, la tercera etapa está relacionada con la aplicación de la propuesta metodológica y la recolección de los datos de la investigación y la última etapa se constituye en la consolidación de los datos y el análisis de los resultados. Fue adoptada como estrategia de validación empírica de la propuesta metodológica a su aplicación en un caso de estudio, efectuando el diagnóstico organizacional de una entidad activa del sector público de la esfera gobernamental del nivel estadual de Brasil, 
teniendo como público objetivo los agentes tomadores de decisión, con fin de identificar el nivel de alineación organizacional cuanto a la Gestión del Conocimiento.

Palabras clave: Evaluación estratégica, Gestión de Conocimiento, Indicadores.

\begin{abstract}
In an attempt to fill an important gap in the field of knowledge management in small and mediumsized organizations, we present a proposal for assessing the level of organizational alignment for the adoption of Knowledge Management as an organizational strategic managerial element, derived from the OKA method. The research was carried out in four stages: the first two involved systematic literature review and documentary research for the formulation of the method proposal and also the selection of the entity to be studied in the case study, the third step is related to the application of the proposal methodological and data collection of the research and the last step consisted in the consolidation of the data and analysis of results. The empirical validation strategy of the methodological proposal was adopted for its application in a case study, carrying out the organizational diagnosis of an active entity in the public sector of the governmental sphere at the state level in Brazil, with the target audience of decision-making agents, with purposes of identifying the level of organizational alignment with regard to Knowledge Management.
\end{abstract}

Keywords: Strategic Evaluation, Knowledge Management, Indicators.

\title{
1 INTRODUCCIÓN
}

En la contemporaneidad es innegable la importancia que el conocimiento presenta para los aspectos relacionados con acciones de la estructura de gestión estratégica de los recursos de capital humano, tangible e intangible, cada vez más desafiada a enfrentar las limitaciones de orden presupuestario, política y administrativa, con vista a la obtención de resultados alineados con las demandas sociales y sujetas a las más diversas adversidades.

La Gestión del Conocimiento ha buscado afirmarse en ese contexto caudaloso como estrategia de acción y campo de investigación, tratando de la problemática de cómo el conocimiento puede venir a ser creado, almacenado, compartido y diseminado en las organizaciones, colocándose junto a otras prácticas de gestión, de manera sistémica y estratégica para posibilitar el uso más efectivo de activos del conocimiento. Entre las prácticas y técnicas adoptadas se destacan aquellas que tratan de la gestión de la innovación, de la educación corporativa, de la gestión por competencias y de su articulación con usos y recursos de las tecnologías de la comunicación y la información.

En el campo de la administración brasileña, en particular de la gestión pública, la gestión del conocimiento ha sido blanco de estudios, investigaciones y aplicaciones prácticas, mereciendo atención las acciones que orbitan de forma germinal al Comité Técnico de Gestión de Conocimiento y de Información Estratégica (CT-GCIE), componente del Comité Ejecutivo del Gobierno Electrónico (CEGE), que lanzó las bases para la creación de una política nacional de gestión del conocimiento, 
homologando y adoptando la metodología OKA - Organizational Knowledge Assessment (Fresneda, 2008).

Para Fonseca (2009), Gestión del Conocimiento (GC) "es un principio gerencial que permite ampliar información, conocimiento, experiencia e intuición en las organizaciones con el fin de generar valor“. Para la autora, la Gestión del Conocimiento es la base de la ventaja competitiva de las organizaciones, a través de la adaptación continua, buscando la innovación y la permanencia en su área de actuación.

Autores como Davenport y Prusak (2003), abordaron la Gestión del Conocimiento como un proceso que es compuesto de diversas etapas intermediarias para su consecución, donde el conocimiento debe ser creado, codificado, distribuido y utilizado en las organizaciones. Para ellos el conocimiento debe ser tratado como un activo organizacional, tan importante en el éxito como los activos económicos o de producción.

\footnotetext{
El conocimiento no es algo nuevo. Nuevo es reconocer el conocimiento como un activo corporativo y entender la necesidad de administrarlo y rodearlo del mismo cuidado dedicado a la obtención de valor de otros activos más tangibles. La necesidad de extraer el máximo de valor del conocimiento organizacional es mayor ahora que en el pasado (Davenport y Prusak 2003).
}

Iniciando una nueva corriente de análisis, Ian Watson (2003) hace una revisión en el concepto de Gestión de Conocimiento buscando una definición restrictiva al gerenciamiento del conocimiento en sí, no despreciando la necesidad de la creación de una estructura organizacional que sea favorable a la creación del conocimiento, sin embargo, centrándose en la adopción de una metodología para la implementación de soluciones de Gestión de Conocimiento.

“Gestión de Conocimiento se refiere a la adquisición, almacenamiento, recuperación, aplicación, generación y revisión de los activos de conocimiento de una organización de manera controlada" (Watson, 2003).

Para Watson (2003) un importante concepto relacionado al conocimiento es el reconocimiento de patrones. El conocimiento no se forma de manera aislada, individual y estática, antes ocurre de manera dinámica, a lo largo de un contínuo, a través del reconocimiento de padrones y su relación con el contexto. Éstas relaciones son observadas en la figura de abajo. 


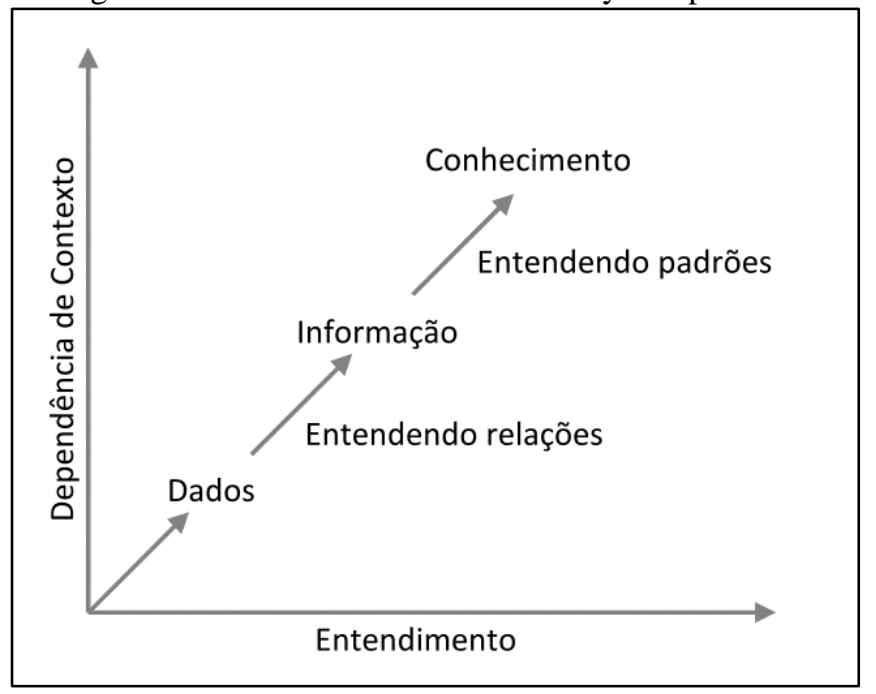

Fuente: Adaptado de Watson (2003).

\section{EL MÉTODO PROPUESTO - ADAPTACIÓN DEL OKA}

La metodología de la Evaluación de Conocimiento Organizacional (OKA- Organizational Knowledge Assessment) fue concebida para evaluar la capacidad y el nivel de preparación de una Organización en la utilización adecuada de sus activos de conocimiento, considerando tres elementos organizacionales como básicos para su aplicación: personas, procesos y sistemas.

Estos elementos a su vez están descompuestos, subdivididos en 14 dimensiones de Conocimiento, que a su vez, son compuestas por métricas que son muy bien definidas en los elementos formadores del método original y reflexionados en los instrumentos de recolección de datos, originalmente compuesto de 187 preguntas (Fonseca, 2009).

En nuestra propuesta de metodología, para que sea posible su uso en el contexto de pequeñas y medianas empresas, es necesario que la complejidad del método sea reducida, adaptando inclusive los elementos finales y también los instrumentos de recolección a la realidad que es presente en las pequeñas y medianas entidades, donde la multiplicidad de papeles ejecutados por los mismos agentes, la ausencia de políticas institucionales de amplio espectro y, a veces, la propia capacidad de acción de planeación estratégica, no hace parte del conjunto de prácticas actuales.

Así, después de estas consideraciones, presentamos las dimensiones a ser consideradas en nuestro método de evaluación, en una visión simplificada, más manteniendo la naturaleza original y la esencia de evaluación de alineación, las cuales están expuestas en la tabla 01, de acuerdo con los ajustes que proponemos en este trabajo de investigación. Fueron utilizadas para nuestra propuesta método 12 de las 14 dimensiones propuestas originalmente en el OKA, para así permitir la evaluación de la alineación también por parte de pequeñas y medianas empresas o entidades a la Gestión de Conocimiento, como un diagnóstico preciso y eficiente para guiar su acción. 
Figura 02 - Método OKA - Estructura e dimensiones

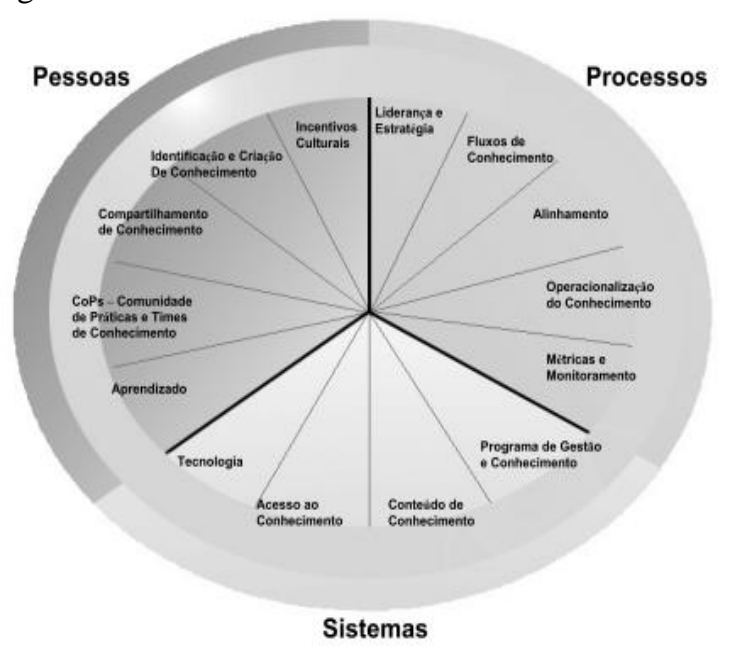

Fuente: Fonseca (2009).

Tabla 1 - Dimensiones del Método OKA

Método OKA - Dimensiones en una visión simplificada

\begin{tabular}{l|l}
\hline \multicolumn{1}{|c|}{ Dimensión Analizada } & \\
\hline Pessoas & L \\
PCI - Cultura \& Incentivos & i \\
\hline & c \\
\hline
\end{tabular}

PKIC - Creacióno e identificción de Conocimiento

\begin{tabular}{l}
\hline PKS - Intercambio de \\
Conocimiento \\
\hline PCPKT - Comunidades de \\
Práctica y Equipos de \\
Conocimiento
\end{tabular}

\section{$\boldsymbol{P K L}$ - Conocimiento \& Aprendizaje}

\section{Processos}

$\boldsymbol{R} \boldsymbol{L}$ - Liderazgo \& Estrategias

$\boldsymbol{R} \boldsymbol{K} \boldsymbol{F}$ - Flujo de Conocimiento
$\boldsymbol{R O K}$ - Operacionalización de Conocimiento

$\boldsymbol{R A}$ - Alineación
Los aspectos culturales implícitos e implícitos, creencias e incentivos que existen dentro de las organizaciones para formatear, crear y dar soporte al uso de activos intelectuales (incluido el conocimiento) para lograr sus objetivos.

La capacidad de las organizaciones y sus "stakeholders" para identificar y crear conocimiento (y otros activos intelectuales), especialmente aquellos que contribuyen a los objetivos de la organización.

La capacidad de las organizaciones y sus "stakeholders" para compartir activos intelectuales de una manera que permita a la empresa alcanzar sus objetivos.

La existencia, naturaleza y uso de grupos de personas que pueden movilizarse eficazmente para resolver problemas y permitir que la organización logre sus objetivos.

La capacidad de la organización para desarrollar su capital humano a través de la formación y otras estructuras o actividades formalmente dirigidas al desarrollo del conocimiento.

El uso de técnicas de GC como modelo de gestión para los líderes y gerentes de la organización.

La naturaleza y capacidad del flujo de conocimiento y otros activos intelectuales dentro de la organización. Incluye la captura, el almacenamiento, la difusión y otros aspectos de la distribución del conocimiento.

La capacidad de la organización para integrar y aplicar el conocimiento dentro de su negocio y procesos operacionales (incluido el desarrollo de nuevos productos, marketing y otros). Representa el ciclo interactivo del conocimiento dentro de los procesos críticos de la organización y, en consecuencia, sus resultados.

El grado en que el objetivo del Programa de Gestión del Conocimiento y su resultado intentan satisfacer o lograr los objetivos y metas de la organización.

Sistemas

SKMTI-Infraestructura

Tecnológica de GC

La capacidad y existencia de infraestructura tecnológica que permita la gestión del conocimiento y el intercambio de mejores prácticas. 


\begin{tabular}{l|l} 
SKAI - Acceso a Infraestructura & $\begin{array}{l}\text { La capacidad e infraestructura existente que permite el acceso e } \\
\text { interacción de los "stakeholders" con los "activos intelectuales" de la } \\
\text { empresa (ya sean sistemas u otras personas). }\end{array}$ \\
\hline $\boldsymbol{S C M}$ - Gestión de Contenido & $\begin{array}{l}\text { El tipo de contenido y las herramientas de gestión de información } \\
\text { que la organización produce y gestiona. }\end{array}$ \\
\hline Critérios Adicionais & $\begin{array}{l}\text { Se considerarán algunos aspectos demográficos de la organización } \\
\text { debido a su relevancia en la interpretación de otros temas. }\end{array}$ \\
\hline DF - Localización & Fuente: Adaptado de Fonseca, et al (2009).
\end{tabular}

Las dimensiones del método se ajustan a la realidad de las organizaciones PMP (Pequeño y Mediano Porte), resultando en la construcción del cuestionario aplicado con un total de 87 (ochenta y siete) preguntas, las cuales buscaban atender las 12 (doce) dimensiones de análisis que componen nuestro escenario para presentar la alineación organizacional.

Con relación al escenario de análisis, dentro del cuerpo funcional de la organización objetivo, se debe buscar como público objetivo para el estudio de caso, los agentes que posean alguna relación funcional directa con la alta gestión, en la posición de staff o comité de gestión, grupos de planeación y de proyectos vinculados con la alta gestión (directores) y que ocupen cargos de gerencia. Se infiere que de esta forma la investigación alcanzará los formadores de opinión y tomadores de decisión, sea por la notoria capacidad técnica o por las propias atribuciones.

Finalmente, considerando el enfoque buscado y para permitir un análisis objetivo en que las dimensiones estén articuladas aún escala que permite el diagnóstico de alineación de la organización, con relación a procesos, personas y sistemas, cada pregunta recibió puntuaciones y fueron sometidas a normalización, en una escala de cero a 10, para normalizar los resultados obtenidos, donde el valor 0 representa ausencia total o desalineación a GC y 10 representa un grado máximo total de alineación de la dimensión analizada a la Gestión de Conocimiento por parte de la organización (Brandalise, 2005).

\section{DIAGNÓSTICANDO LA GESTIÓN DE CONOCIMIENTO - VALIDACIÓN DEL MÉTODO}

La entidad analizada es una autarquía especial, integrante de la Administración Indirecta del Poder Ejecutivo del Estado de Sergipe, responsable por la ejecución de las políticas relacionadas con el medio ambiente, dentro del estado de Sergipe:

Ejecuta dentro de su área de actuación las siguientes acciones:

- Acompañamiento, control y fiscalización de las transformaciones ocurridas en el medio ambiente, hacia la realización de cambios ecológicos y control de la contaminación. 
- Elaboración de normas y padrones relativos a la preservación del medio ambiente y el control de la contaminación.

- Ejecución de programas de entrenamiento para la formación y perfeccionamiento de técnicos y especialistas.

- Desarrollo de programas de divulgación, con el objetivo del uso adecuado de los recursos naturales, preservación del medio ambiente y el control de la contaminación.

Entre las diversas áreas involucradas en la gestión ambiental, desarrolla las siguientes actividades:

- Protección de los recursos naturales.

- Diagnóstico de las condiciones de residuos sólidos en el Estado de Sergipe.

- Monitoreo y evaluación de la calidad ambiental.

- Licenciamiento ambiental.

- Análisis de estudios ambientales (Estudio de Impacto Ambiental - EIA /Relatorio de Impacto Ambiental - RIMA y Relatorio de Control Ambiental - RCA).

- Diagnóstico y fiscalización ambiental.

\section{RESULTADOS Y DISCUSIÓN}

El Público Objetivo fue definido adoptando los siguientes criterios: servidores que tuvieran una relación funcional directa con la alta gestión; ocupantes de cargos de asesoramiento o comités de gestión; participante de grupos de planificación o de proyecto y los ocupantes de cargos de gerencia. Esta selección llevó a una muestra de investigación de 25 (veinticinco) servidores, generando el envío de 25 (veinticinco) invitaciones para participar de la investigación.

Gráfico 2 - Distribución jerárquica del público objetivo

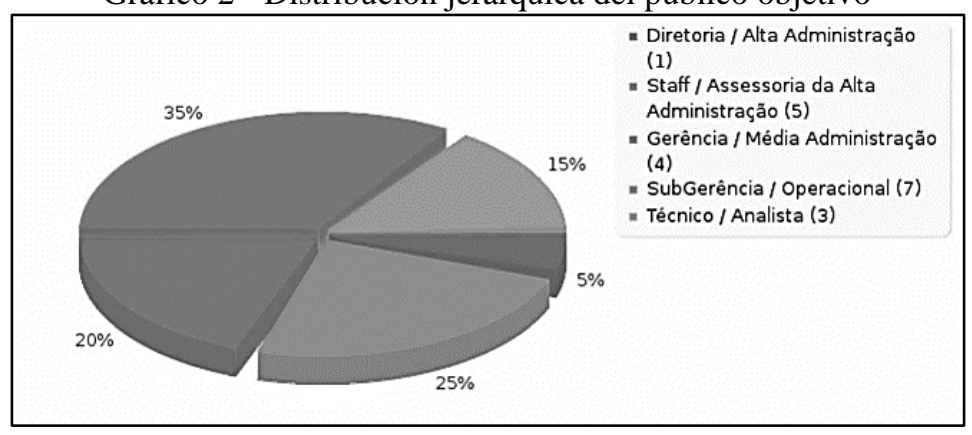

Fuente: elaborado por los autores. 
El cuestionario se pudo responder tanto electrónicamente por acceso al sistema disponible en Intranet, así como en su formato impreso. Como se trató de un cuestionario extenso, contando con 87 preguntas, percibimos la necesidad de nivelación de conocimientos, por tanto, efectuamos una sensibilización y nos dispusimos para ofrecer apoyo individual a los encuestados.

La Tabla 2 recoge los resultados obtenidos y generados con los dados recolectados, después de tabulación y normalización:

Tabla 2: Resultado del cuestionario aplicado

\begin{tabular}{l|l|l} 
Elemento & Dimensión & Valor \\
\hline \multirow{5}{*}{ Personas } & Conocimiento y Aprendizaje & $\mathbf{4 , 1 4}$ \\
\cline { 2 - 3 } & Intercambio de Conocimiento & $\mathbf{5 , 7 7}$ \\
\cline { 2 - 3 } & $\begin{array}{l}\text { Comunidades de Práctica y Equipos de } \\
\text { Conocimiento }\end{array}$ & $\mathbf{8 , 7 8}$ \\
\cline { 2 - 3 } & Creación e Identificación del Conocimiento & $\mathbf{5 , 7 5}$ \\
\cline { 2 - 3 } & Incentivos Culturales & $\mathbf{3 , 8 4}$ \\
\hline \multirow{5}{*}{ Procesos } & Alineamiento & $\mathbf{5 , 7 0}$ \\
\cline { 2 - 3 } & Flujo do Conocimiento & $\mathbf{5 , 7 3}$ \\
\cline { 2 - 3 } & Liderazgo y Estrategia & $\mathbf{4 , 7 9}$ \\
\cline { 2 - 3 } & Operacionalización del Conocimiento & $\mathbf{4 , 1 6}$ \\
\hline \multirow{5}{*}{ Sistemas } & Contenido del Conocimiento & $\mathbf{5 , 8 2}$ \\
\cline { 2 - 3 } & Infraestructura de Acceso al Conocimiento & $\mathbf{3 , 5 7}$ \\
\cline { 2 - 3 } & Tecnología & \\
\cline { 2 - 3 } & & \\
\cline { 2 - 3 } & & \\
& &
\end{tabular}

Fuente: Elaborada por los autores.

A continuación, presentamos nuestro análisis de los datos recolectado, con base en las respuestas, así como en el análisis documental y en la perspectiva de participantes activos en la gestión.

El Elemento Sistemas presentó notas por debajo de la media en la evaluación de los investigados, en que se destaca la baja nota atribuida a la dimensión Tecnología. Un análisis posible a este escenario es que la capacidad y existencia de infraestructura tecnológica con fines específicos de gestión del conocimiento presentan un grado de alineación que necesita atención urgente, en caso se decida por la implantación de estas prácticas, ya que la gestión del conocimiento es muy dependiente de recursos de tecnología de la información y comunicación.

El Elemento Personas consiguió la mayor puntuación dimensional de la institución analizada, con 8,78 referente a las Comunidades de Práctica y Equipos de Conocimiento. Lo que significó que, a pesar de no existir un programa institucional de gestión del conocimiento, las interacciones entre los equipos e individuos ocurren, aunque sea informalmente, lo que genera una red de interacciones 
personales a partir de intereses en común, que la coloca como una dimensión de alto potencial estratégico y alineada con las prácticas de gestión del conocimiento.

En cuanto a la dimensión Incentivos Culturales, la baja puntuación verificada está relacionada, primeramente, con el hecho de no existir en la organización una política establecida de incentivo a la convivencia, ni siquiera de forma informal, entre las personas que poseen intereses comunes, de modo que el desarrollo, ampliación e intercambio de conocimiento sea recompensado.

El resultado del Elemento Procesos, con la relación a las dimensiones Liderazgo y Estrategia, así como Alineamiento, presentan un valor positivo, lo que demuestra un buen grado de accesibilidad, principalmente, a los gerentes y a la alta gerencia.

Como agencia pública, la vinculación a metas y resultados forma parte de la realidad de la organización; con el resultado demostrado en el ítem de conocimiento, hay un buen nivel de alineación de las prácticas institucionales o, en otras palabras, entre el conocimiento disponible y las metas organizacionales.

Gráfico 5: Gráfico SPIDER del resultado del cuestionario aplicado

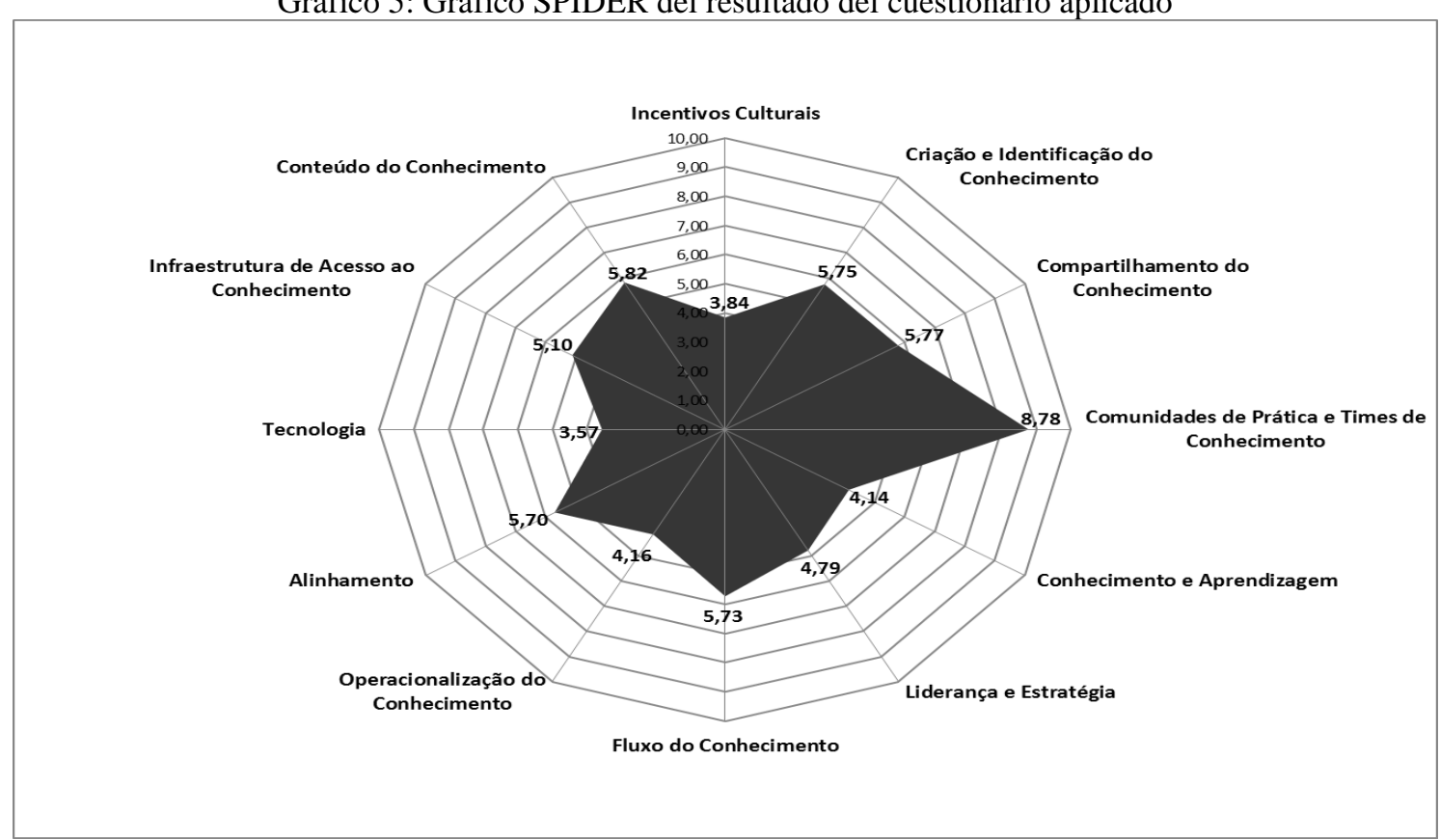

Fuente: Elaborado por los autores.

Los valores de las dimensiones Flujo y Operacionalización del Conocimiento presentan una gran relación de dependencia con el Elemento Sistemas, por tanto, se puede inferir que su bajo desempeño esté relacionado con la deficiencia apuntada en aquel Elemento. Sin embargo, las dimensiones reducidas de la organización y la facilidad de acceso a los poseedores de conocimiento, permiten que nuevos proyectos y prácticas de innovación acontezcan, incluso sin el apoyo tecnológico adecuado. 
De la misma forma, el Intercambio de Conocimiento presenta un grado positivo en el universo de análisis, lo que demuestra que la muestra del cuerpo funcional que respondió al elemento de recolección de datos, tiene una mentalidad dirigida a compartir sus conocimientos, sin manifestar miedo aparente de perder su importancia dentro de la organización. Podemos además asociar ese resultado al grado de formación y nivel educacional formal del grupo investigado, ya que existe en la institución un grado de especialización y de acciones individuales bien definidas para áreas específicas de actividad, hecho demostrado con los resultados demográficos, que colocan $95 \%$ de los servidores investigados con grado de formación de nivel superior, y $45 \%$ con nivel de pos-graduación, de acuerdo con datos obtenidos de las preguntas demográficas incluidas en el instrumento de investigación.

Con un resultado de 4,14, la dimensión Conocimiento y Aprendizaje necesita de atención. Como resultado de la investigación documental asociada a resultados del cuestionario aplicado, se verificó que la organización no posee un plan de desarrollo de personas que busque desarrollar las habilidades de su cuerpo técnico. También no existe un programa de incentivo a la innovación o al intercambio de buenas prácticas. Si se considera que el cuerpo técnico tiene un grado de educación formal elevado, este valor puede significar una potencial perdida de talentos por parte de la organización.

Al efectuar un análisis relacionada con los Elementos Sistemas, Procesos y Personas, es necesaria una atención especial con las dimensiones de Sistemas, debido a que sus resultados demuestran que existe poco soporte para las acciones de gestión del conocimiento. En cuanto a Procesos, su dependencia tecnológica también influenció negativamente en los resultados. Mientras que el Elemento Personas, aún sin apoyo institucional declarado, consiguió alcanzar valores por encima de la media, ubicándose como elemento base para posibles iniciativas de gestión del conocimiento en el ámbito de la Administración Estadual del Medio Ambiente.

\section{CONCLUSIÓN}

Previamente, y de forma independiente a la solución de mercado o de la metodología electa para la implementación de proyectos de Gestión del Conocimiento en una organización, es necesario visualizar el grado de alineación organizacional con relación a la Gestión del Conocimiento, previo a la decisión de adoptar alguna política estratégica de gestión del conocimiento organizacional. Ese diagnóstico inicial debe responder a las preguntas sobre qué prácticas de captura, intercambio e innovación ya existen; cuales deben ser creadas y a qué ritmo, para la implementación de la Gestión del Conocimiento.

La verificación del nivel de alineamiento con la gestión del conocimiento, a través de la aplicación de una adaptación del Método OKA, como fue propuesto; además de la posibilidad de 
acompañamiento del proceso de implementación y del cuadro de indicadores presentados, fomentará la temática en la alta gestión de la organización y generará los subsidios de capital social y apoyo necesario para la adopción de medidas que permitan la construcción de una plano de implementación de gestión del conocimiento, apoyado por los stakeholders correctos.

Por tanto, después de haber utilizado nuestro método propuesto a partir de una adaptación del Método OKA, y efectuada su aplicación en una organización de tamaño mediano, para verificar su grado de alineación a la gestión del conocimiento, presentamos como resultados inmediatos de la aplicación de la metodología:

- Un amplio potencial para que se torne un instrumento de diagnóstico de situación de la Gestión del Conocimiento para entidades de pequeño y mediano tamaño.

- Se logró efectivamente evaluar el grado de alineación institucional con la Gestión del Conocimiento, considerando las dimensiones y métricas propuestas.

- Contribuyó a fomentar la discusión acerca de la Gestión del Conocimiento en el ámbito del equipo organizacional.

Desde un punto de vista gerencial, el diagnóstico situacional de la institución permitió evidenciar cuales son y donde están las fortalezas y debilidades de sus recursos de conocimiento, información que fue encaminada a los gestores en términos de retroalimentación por la investigación, que podrá ser utilizada como base para la generar o actualizar planes de acción en el ambiente institucional, con enfoque a la gestión del conocimiento.

Ganancias indirectas con la aplicación de nuestra propuesta metodológica, evidentemente considerando las restricciones particulares al caso en estudio, pueden ser obtenidos a través de la identificación de elementos disonantes en la estructura administrativa, ajustando el enfoque de las inversiones, priorizando aquellas áreas que necesitan de mayor atención. Al mismo tiempo y de manera complementar, las prácticas positivas pueden ser localizadas e incentivadas, lo que permite la creación de un circulo virtuosos de gestión del conocimiento, impactando en la cantidad y calidad de la adquisición, mantenimiento e intercambio de activos intelectuales.

Finalmente, entendemos que nuestra propuesta metodológica de evaluación del nivel de alineamiento organizacional para la adopción de la Gestión del Conocimiento, discutida y ejecutada empíricamente de manera exploratoria, se mostró viable e con potencial para que se torne un instrumento adecuado para efectuar un diagnóstico de la Gestión del Conocimiento en entidades de pequeño y mediano tamaño, entregando a los actores, que se involucren en la temática, una visión privilegiada de la realidad institucional con relación a su alineamiento a la Gestión del Conocimiento. 


\section{REFERENCIAS}

BRANDALISE, L. T. (2005). Modelo de medição de percepção e comportamento: uma revisão. Brandalise, Loreni \& Bertolini, Geysler. (2013). INSTRUMENTOS DE MEDIÇÃO DE PERCEPÇÃO E COMPORTAMENTO - UMA REVISÃO. Rev. Ciênc. Empres. UNIPAR. 14. 7-34.

DAVENPORT, T. H.; PRUSAK, L. (2003) Conhecimento empresarial: como as organizações gerenciam o seu capital intelectual. Rio de Janeiro: Elsevier, $14^{\mathrm{a}}$ Edição.

FONSECA, A. F. TORRES, M. F. P. GARCIA, J. C. R. (2010). Definição de Referências e Adequação do Uso do Método OKA (Organizational Knowledge Assessment) na Medição dos Elementos Necessários para Gestão do Conhecimento em Organizações de Pequeno e Médio Porte.Segundo Simposio Iberoamericano em Generación, Comunicación y Gerencia del Conocimiento: GCGC 2010, Orlando, Florida, USA.

FONSECA, A. F. (2006). Organizational Knowledge Assessment Methodology. World Bank Institute. Washington, D.C.

FRESNEDA, P S. GONÇALVES, S. M. G. (2008) Diretrizes orientadoras para a implantação da Gestão do Conhecimento na Administração Pública Federal. In: CONSAD - Diretrizes orientadoras de GC na APF, 25 abril de.

GONÇALVES, S. M. G.; PAPA, M.; FONSECA A. F. (2008). Diagnóstico Da Gestão Do Conhecimento Nas Organizações Públicas Utilizando O Método Organizational Knowledge Assessment (Oka). II Congresso Consad de Gestão Pública

HAIR, J. F. (2006). Fundamentos de métodos de pesquisa em administração. Porto Alegre: Bookman. (Reimpressão)

PROBST, G.; RAUB. S. ROMHARDT, K. (2002) Gestão do conhecimento: os elementos construtivos do sucesso. Porto Alegre: Bookman.

SOUZA, B. B.; MELO, J. D.; CORREA, M., MOURA, A. O., SANTOS, F. F., GUIMARÃES, M. C.; BARROS NETO, H. M. C. (2016). Sensoriamento remoto aplicado ao mapeamento e quantificação de áreas de manguezal no estado de Sergipe. Caminhos de Geografia, 17(57), 126-134.

WATSON, I. D. (2003) Applying knowledge management: techniques for building corporate memories. Morgan Kaufmann, San Francisco. 